\title{
Safety and Efficacy of Selective Intra-arterial Thrombolysis for Central Retinal Artery Occlusion
}

\author{
Sang Jun Ko, In Choel Shin ${ }^{1}$, Dae Won $\mathrm{Kim}^{2}$, Si Sung $\mathrm{Choi}^{3}$, Yun Sik Yang ${ }^{1}$ \\ ${ }^{1}$ Department of Ophthalmology, Wonkwang University College of Medicine, Iksan, Korea \\ ${ }^{2}$ Department of Neurosurgery, Wonkwang University College of Medicine, Iksan, Korea \\ ${ }^{3}$ Department of Radiology, Wonkwang University College of Medicine, Iksan, Korea
}

Purpose: The purpose of this study was to determine the efficacy and safety of selective intra-arterial thrombolysis in patients with central retinal artery occlusion (CRAO).

Methods: Medical records for 44 eyes of 44 patients diagnosed with acute non-arteritic CRAO and thrombolysis between October 2010 and February 2019 were analyzed retrospectively. Based on visual acuity, fundoscopic findings, and fluorescein angiography, the patients were classified into three stages: incomplete, subtotal, and total. The perfusion state using the best-corrected visual acuity (BCVA), arm to retina time, and arteriovenous passage times, after 1 month, 6 months, and at the final visit after the procedure, were compared with baseline readings.

Results: Improvement of visual acuity was confirmed in 31 out of 44 patients (70.45\%). The mean BCVA of 44 patients changed from $1.65 \pm 0.78$ logarithmic minimum angle of resolution ( $\log M A R$ ) at the first visit to $1.18 \pm 0.91 \log M A R$ at the last visit $(p=0.114)$. The BCVA according to CRAO stage was $0.08 \pm 0.11 \log$ MAR for the incomplete stage at the first visit, 0.06 $\pm 0.05 \log$ MAR $(p=0.933) 1$ month after the procedure, and $0.05 \pm 0.07 \log$ MAR $(p=0.933)$ at the last visit. In the subtotal stage, the results were $1.81 \pm 0.54 \log M A R$ at the first visit, $1.63 \pm 0.76 \log$ MAR $(p=0.035) 1$ month after the procedure, and $1.36 \pm 0.85 \log$ MAR $(p=0.014)$ at the last visit. For the total stage of BCVA, the result at the first visit was $2.36 \pm 0.25 \log M A R$, and it was $2.30 \pm 0.30 \operatorname{logMAR}(p=0.510) 1$ month after the procedure, and $2.42 \pm 0.30 \log \operatorname{MAR}(p=0.642)$ at the last visit. Reperfusion was observed in 40 patients out of the 44 (90.91\%).

Conclusions: Selective intra-arterial thrombolysis can be helpful in patients with subtotal CRAO in terms of visual improvement and retinal arterial reperfusion.

Key Words: Retinal artery occlusion, Thrombolytic therapy, Visual acuity

Central retinal artery occlusion (CRAO) is an ophthalmic emergency caused by the interruption of blood supply

Received: June 15, 2020 Final revision: May 10, 2021

Accepted: May 18, 2021

Corresponding Author: Yun Sik Yang, MD, PhD. Department of Ophthalmology, Wonkwang University Hospital, 895 Muwang-ro, Iksan 54538, Korea. Tel: 82-63-859-1370, Fax: 82-63-855-1801, E-mail: ysyang@ wonkwang.ac.kr to the inner layer of the retina, subsequently leading to a sudden painless loss of vision [1]. Approximately $80 \%$ of patients experience a $20 / 400$ or worse vision [2], and CRAO is known to occur in approximately one to two per 100,000 people [3].

CRAO, the ocular analog of cerebral ischemic stroke, arises due to the occlusion of the central retinal artery (CRA) [4]. It has been associated with a high risk of cere- 
bral infarction as well as heart disease [5] and is considered to be an ophthalmic and medical emergency [6]. Although many evidence-based protocols have been used in the management of stroke [7], there are no clear guidelines for the treatment of acute CRAO, as it is still the subject of deliberations in the field [8]. Moreover, natural reperfusion of occlusion occurs in $8 \%$ to $25 \%$ of the cases [5,9]. Conservative treatments, also known as "the standard," are usually used to treat CRAO. They consist of globe compression, anterior chamber paracentesis, administration of anticoagulants and vasodilators, inhalation of a carbogen, and administration of methylprednisolone. However, none of these showed any results that were significantly better than those obtained with the placebo [8-14]. Therefore, injecting a thrombolytic agent directly into the occluded vessel (intra-arterial thrombolysis, IAT) could be considered as a therapeutic option for CRAO. Moreover, its effectiveness has been confirmed in several retrospective cases as well as cohort studies [15-19].

The European Assessment Group for Lysis in the Eye (EAGLE) trial, a prospective, randomized, and multicenter study, compared the effects of IAT with that of the standard conservative therapy. There were no significant differences between the final best-corrected visual acuity (BCVA) of the two therapies, although there were adverse reactions that occurred more frequently in the IAT group. Therefore, IAT was not recommended for the treatment of CRAO [20,21]. Moreover, since thrombolytics have been used to treat acute cerebral infarction, and as CRAO is also considered as the ocular analog of infarction, it could be logical to consider an intravenous or intra-arterial injection of thrombolytics as one of its treatment methods. Although IAT has not been recommended for the treatment of CRAO in the EAGLE trial due to certain limitations, such as the small number of participants and no broad or selective IAT being conducted in consideration of the treatment window, it may not be reasonable to exclude IAT as a treatment modality. Therefore, further studies on its efficacy, safety, and treatment window are necessary $[3,5,22]$.

CRAO can be divided into three stages based on visual acuity (VA), fundoscopic findings, and fluorescein angiography (FA), which helps determine its prognosis. Incomplete CRAO shows a decrease in VA, mild retinal edema, a slightly macular cherry-red spot, and a mildly delayed flow in FA. Subtotal CRAO shows a severe decrease in VA and macular cherry-red spot with marked retinal edema and a
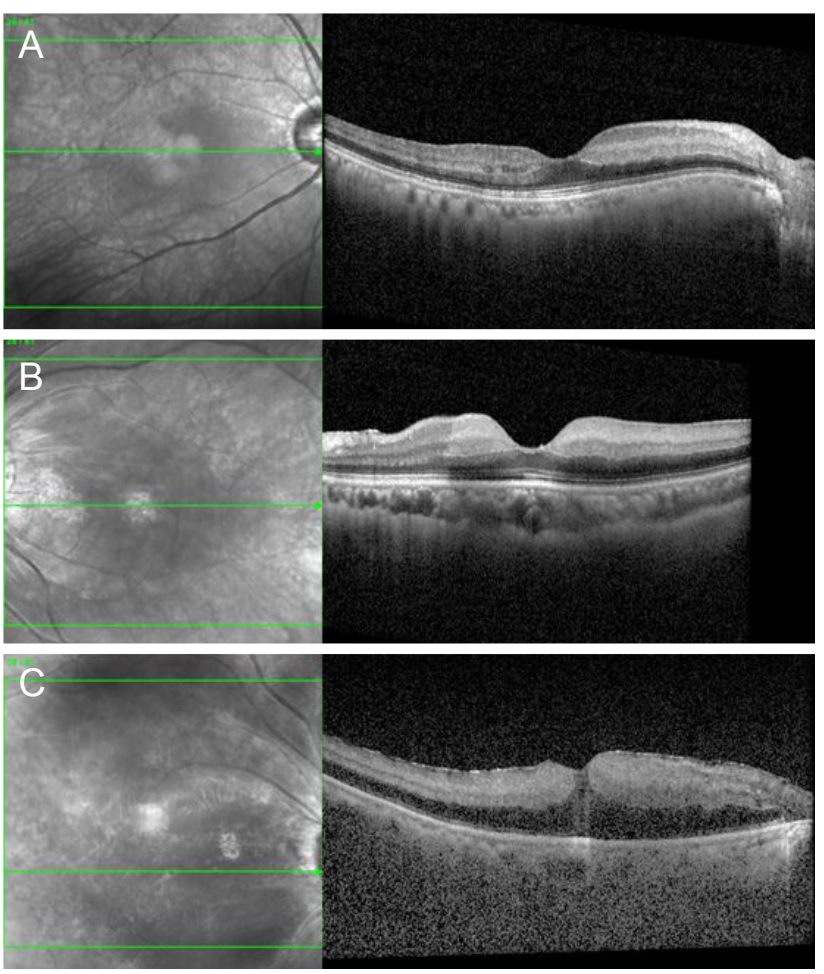

Fig. 1. The initial optical coherence tomography of three central retinal artery occlusion (CRAO) subtype group. (A) Incomplete CRAO, (B) subtotal CRAO, and (C) total CRAO.

significant flow decrease in FA. Total CRAO shows massive retinal edema, with VA dropping to no light perception, along with choroidal hypoperfusion and, in some instances, no cherry-red spot [22-25] (Fig. 1A-1C). When compared to the PROACT II study, the selective IAT conducted in our institution was performed as close to the ophthalmic artery as possible.

Therefore, to evaluate the efficacy and safety of selective IAT, we retrospectively studied patients who were classified into groups according to their CRAO stage based on test results. We then compared BCVA and evaluated the change in the retinal perfusion state by using FA. Furthermore, we noted the adverse reactions of IAT.

\section{Materials and Methods}

\section{Study design}

This study retrospectively analyzed the medical records of patients who were diagnosed with acute non-arteritic $\mathrm{CRAO}$ and underwent thrombolysis at the neurosurgery or 
Table 1. Baseline characteristics of 44 patients who underwent intra-arterial thrombolysis for CRAO

\begin{tabular}{|c|c|c|c|c|c|}
\hline Variable & Data (all) & Incomplete CRAO & Subtotal CRAO & Total CRAO & $p$-value \\
\hline Total (eye, \%) & 44 & $5(11.36)$ & $34(77.28)$ & $5(11.36)$ & $0.296^{*}$ \\
\hline Male & $31(70.45)$ & 5 & 23 & 3 & \\
\hline Female & $13(29.55)$ & 0 & 11 & 2 & \\
\hline \multicolumn{6}{|l|}{ Age (yr) } \\
\hline Mean \pm SD & $67.00 \pm 12.43$ & $56.00 \pm 1.42$ & $67.47 \pm 12.31$ & $74.80 \pm 2.43$ & $0.069^{*}$ \\
\hline Range & $46-89$ & $47-69$ & $46-89$ & $69-83$ & \\
\hline \multicolumn{6}{|l|}{ BCVA (logMAR) } \\
\hline Mean \pm SD & $1.65 \pm 0.79$ & $0.08 \pm 0.11$ & $1.80 \pm 0.54$ & $2.36 \pm 0.25$ & $0.000^{*}$ \\
\hline Range & $0.00-2.60$ & $0.0-0.22$ & $0.16-2.30$ & $2.00-2.60$ & \\
\hline \multicolumn{6}{|l|}{ ART (sec) } \\
\hline Mean \pm SD & $26.70 \pm 14.27$ & $20.80 \pm 8.67$ & $29.03 \pm 19.47$ & $24.20 \pm 6.61$ & $0.504^{*}$ \\
\hline Range & $13-100$ & $13-34$ & $14-100$ & $17-35$ & \\
\hline \multicolumn{6}{|l|}{$\begin{array}{l}\text { Duration of start to IAT } \\
\text { after onset (hr) }\end{array}$} \\
\hline Mean \pm SD & $16.96 \pm 16.93$ & $20.70 \pm 29.80$ & $17.69 \pm 15.73$ & $8.20 \pm 5.77$ & $0.323^{*}$ \\
\hline Range & $0.50-74.50$ & $6.00-74.00$ & $3.00-74.50$ & $0.50-15.50$ & \\
\hline \multicolumn{6}{|l|}{ Underlying diseases } \\
\hline Hypertension & $21(47.73)$ & 1 & 17 & 3 & $0.704^{\dagger}$ \\
\hline Diabetes mellitus & $10(22.73)$ & 0 & 7 & 3 & $0.063^{\dagger}$ \\
\hline Hyperlipidemia & $7(15.91)$ & 1 & 5 & 1 & $0.951^{\dagger}$ \\
\hline Myocardial infarction & $4(9.09)$ & 0 & 3 & 1 & $0.543^{\dagger}$ \\
\hline
\end{tabular}

Values are presented as number $(\%)$.

$\mathrm{CRAO}=$ central retinal artery occlusion; $\mathrm{SD}=$ standard deviation; $\mathrm{BCVA}=$ best-corrected visual acuity; $\log \mathrm{MAR}=\operatorname{logarithmic}$ minimum angle of resolution; ART = arm-to-retinal time; IAT = intra-arterial thrombolysis.

$p$-value for comparison among three subgroups by the "Kruskal-Wallis test and the chi-squared tests according to the results of the Kolmogorov-Smirnov test.

radiology department, after visiting ophthalmology outpatient and the emergency department, from October 2010 to February 2019. This study was approved by the institutional review board of Wonkwang University Hospital (WKUH 2020-04-025-001) and complied with the Declaration of Helsinki. Informed consent was waived due to the retrospective nature of the study using medical records.

\section{Patient characteristics}

Participants of the study included those diagnosed with non-arteritic CRAO and could be followed up for at least 6 months. A total of 44 eyes from 44 patients (31 males, 13 females; mean age, $67.00 \pm 12.43$ years; age range, 46-89 years) were included in this study. The baseline characteristics and exclusion criteria for the participating patients are outlined in Table 1 and 2. In terms of the CRAO staging, there were five patients at the incomplete
Table 2. Exclusion criteria

\begin{tabular}{l}
\hline Criteria \\
\hline Ocular factors \\
Branch retinal artery occlusion \\
Combined central retinal vein occlusion \\
Elevated intraocular pressure \\
Existed ocular problems decreasing visual function \\
General factors \\
Uncontrolled hypertension (systolic blood pressure \\
$>200$ mmHg) \\
Previous history of heart attack, intracranial bleeding or \\
surgery, cerebral infarction \\
Recently treatment with antithrombotic drugs \\
Coagulation disorder \\
Allergic reaction to the contrast agent
\end{tabular}


stage $(11.36 \%), 34$ at the subtotal $(77.28 \%)$, and five at the total stage $(11.36 \%)$.

\section{Examination}

The following information was collected, while medical and ophthalmic examinations were conducted on all patients during their visit to the outpatient and the emergency department: (1) past medical history of conditions such as hypertension, diabetes, hyperlipidemia, cerebrovascular disease, and cardiovascular disease; (2) the time taken from symptom onset to the hospital visit; (3) BCVA using the Snellen chart; (4) intraocular pressure; (5) fundus examination; (6) spectral-domain optical coherence tomography (Spectralis OCT; Heidelberg Engineering, Heidelberg, Germany), and 7) FA (Spectralis HRA2, Heidelberg Engineering).

An adequate explanation was provided to the patient and guardian regarding the necessity, procedural method, side effects, and precautions for thrombolysis. After consent was obtained, thrombolysis was performed at the neurosurgery or radiology department of the hospital. The time taken from the onset of symptoms until the procedure was noted.

In addition, the patients were assessed for any complications that occurred either after the examinations at the time of visit or after the procedure, excluding for OCT; or at their visit 1 month after the procedure; or at their final visit 6 months after the procedure.

\section{IAT}

The procedure was performed as follows: a 6 Fr sheath was inserted through a puncture in the femoral artery, while a $6 \mathrm{Fr}$ (or $5 \mathrm{Fr}$ ) catheter was used to access the common carotid artery and cerebral blood vessels. The ophthalmic artery flow was confirmed through an internal carotid artery (ICA) angiogram, while the ophthalmic artery orifice was observed with an oblique lateral angiogram. This was followed by a confirmation of the collateral ophthalmic artery flow and the occlusion site through an external carotid artery angiogram. After a precise selection of ophthalmic artery using a microwire and catheter, 300,000 units of urokinase (UK) (Grenn Cross, Yongin, Korea) were infused into the artery. If the ophthalmic artery was visible at this stage, the drug was injected into the lesion, and if a precise selection was not possible, the drug was injected near the entrance of the ophthalmic artery.
Finally, we observed the change in ophthalmic artery flow using the ICA angiogram. After completion of the procedure, $100 \mathrm{mg}$ of aspirin (Astrix; Boryung, Seoul, Korea) was administered for maintenance therapy.

\section{Assessment of treatment efficacy}

First, the patient group was classified into three stages (incomplete, subtotal, total) based on VA, fundoscopic findings, and FA. The VA was measured using the Snellen chart and converted to logarithmic minimum angle of resolution $(\log$ MAR) measurement for statistical analysis at a later stage. Low VAs that were obtainable through measures such as counting fingers or hand motion was converted to $\log$ MAR measurements by the method proposed by Lange et al. [26]. Significant improvement in VA was defined as an improvement of $0.3 \log$ MAR or more.

Arm-to-retina time (ART) and arteriovenous passage time (AVP) were measured using FA to identify the problem of perfusion as well as the CRAO stage. After IAT, if ART and AVP showed a decrease of more than 20\%, compared to the readings at the time of the first visit, CRAO was considered to have achieved reperfusion.

\section{Statistical analysis}

For data analysis, PASW Statistics ver. 18 (SPSS Inc., Chicago, IL, USA) was used. This study had a small number of participants. Therefore, to examine the normality of the distributions of the participating groups, the comparison between the two groups, which showed normality according to the results of a Kolmogorov-Smirnov's normality test, was tested using the independent $t$-test. For data that were not normally distributed, assessments were done using the Mann-Whitney test. The Kruskal-Wallis test was used for comparisons between three different groups, and all results were considered to be statistically significant if the $p$-value was less than 0.05 .

\section{Results}

\section{Visual and perfusion outcomes}

VA improvement was confirmed in 31 of 44 patients (70.45\%). The mean BCVA of the 44 patients was $1.65 \pm$ 
A

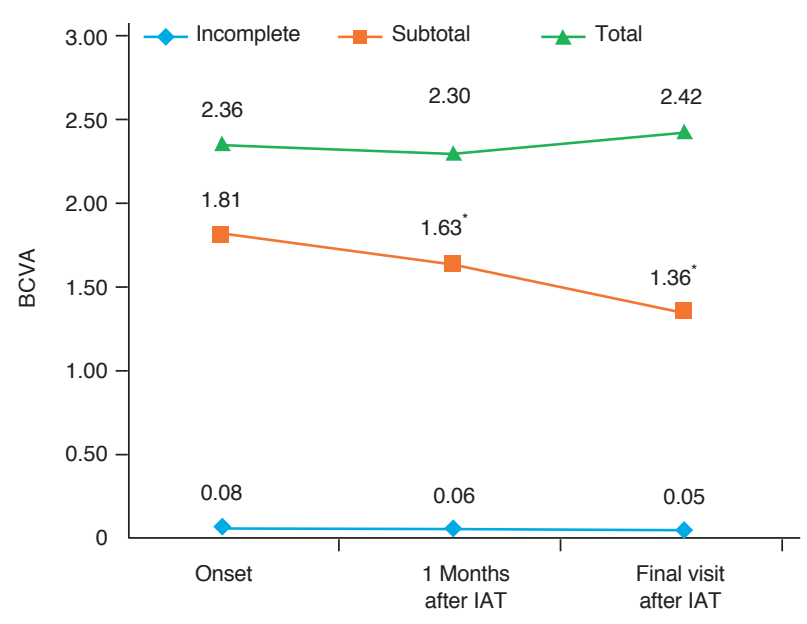

B

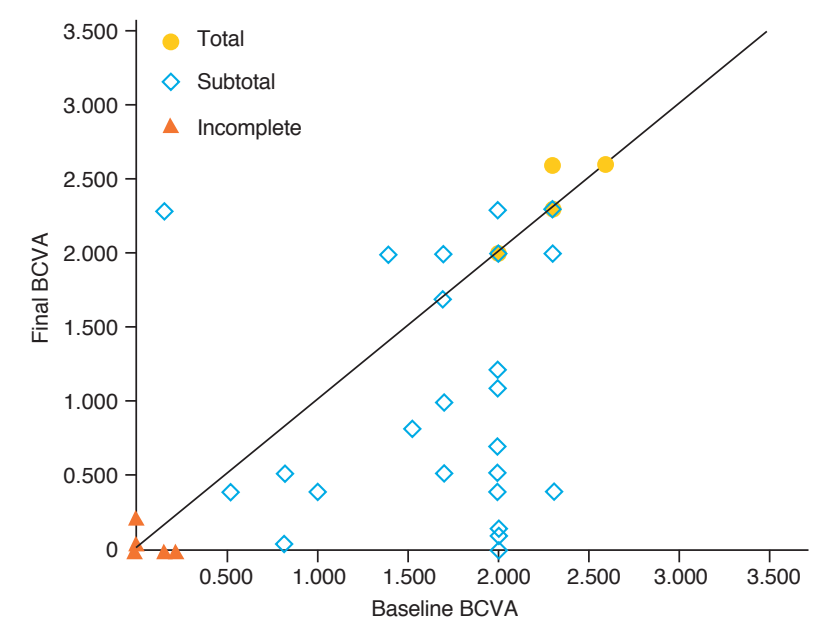

Fig. 2. Comprehensive changes in visual acuity after intra-arterial thrombolysis (IAT) in central retinal artery occlusion patients. (A) Mean logarithmic minimum angle of resolution (logMAR) visual acuity after IAT treatment according to stage of central retinal artery occlusion. (B) Changes in the Final $\log$ MAR visual acuity according to stage of central retinal artery occlusion. BCVA $=$ best-corrected visual acuity. "Statistically significance is $p<0.05$ with baseline.

Table 3. Visual improvement at the last follow-up after intra-arterial thrombolysis therapy according to stage of CRAO

\begin{tabular}{|c|c|c|c|c|}
\hline & Incomplete CRAO & Subtotal CRAO & Total CRAO & $p$-value \\
\hline Total patients & 5 & 34 & 5 & $0.002^{*}$ \\
\hline $\begin{array}{l}\text { Significant visual } \\
\text { improvement }^{\dagger}\end{array}$ & 0 & $17(50)$ & 0 & \\
\hline Partial visual improvement ${ }^{\star}$ & $2(40)$ & $1(2.94)$ & 0 & \\
\hline No visual improvement ${ }^{\S}$ & $3(60)$ & $16(47.06)$ & $5(100)$ & \\
\hline
\end{tabular}

Values are presented as number (\%).

$\mathrm{CRAO}=$ central retinal artery occlusion.

$p$-value for comparison among three subgroups by the * chi-squared test; ${ }^{\dagger}$ Significant visual improvement of $\geq 0.3$ logMAR; ${ }^{*}$ Visual improvement of $<0.3 \log$ MAR; ${ }^{\S}$ No visual acuity change.

$0.78 \log$ MAR at the first visit, $1.375 \pm 0.91 \log$ MAR $(p=$ 0.036) 1 month after the procedure, and $1.18 \pm 0.91$ $\operatorname{logMAR}(p=0.114)$ at the last visit. In terms of BCVA based on CRAO stages, the results for those in the incomplete stage were $0.08 \pm 0.11 \log$ MAR at the first visit, 0.06 $\pm 0.05 \log$ MAR $(p=0.933) 1$ month after the procedure, and $0.05 \pm 0.07 \log$ MAR $(p=0.933)$ at the final visit. For those with subtotal-stage disease, BCVA was $1.81 \pm 0.54$ $\log$ MAR at the first visit, $1.63 \pm 0.76 \log$ MAR $(p=0.035)$ 1 month after the procedure, and $1.36 \pm 0.85 \log \operatorname{MAR}(p=$ $0.014)$ at the final visit. For those with total-stage disease, it was $2.36 \pm 0.25 \log$ MAR at the first visit, $2.30 \pm 0.30 \log$ $\operatorname{MAR}(p=0.510) 1$ month after the procedure, and $2.42 \pm$ $0.30 \log$ MAR $(p=0.642)$ at the final visit. On comparing the results of the three stages, there was a statistical signif- icance for the change in BCVA in only the subtotal stage (Fig. 2A, 2B).

Significant VA improvements of at least $0.3 \log$ MAR were noted in 17 out of 44 patients (38.64\%). VA improvement was less than $0.3 \log$ MAR in three patients $(6.82 \%)$, while 24 patients (54.55\%) experienced no improvement. When examining results by stages, a VA improvement of less than $0.3 \log$ MAR was found in two out of five patients $(40 \%)$ at the incomplete stage, and the remaining three patients $(60 \%)$ did not show an improved VA. Moreover, at the subtotal stage, 17 out of 34 patients (50\%) showed significant improvements, one patient (2.94\%) showed an improvement of less than $0.3 \log$ MAR, and 16 patients $(47.06 \%)$ experienced none. However, in the total stage, all five patients $(100 \%)$ did not show any improvement (Table 3). 
Table 4. Recovery of central retinal artery perfusion after intra-arterial thrombolysis therapy according to the stage of CRAO

\begin{tabular}{lcc}
\hline Stage of CRAO & Reperfusion & No reperfusion \\
\hline Incomplete CRAO & $5(100)$ & 0 \\
Subtotal CRAO & $31(91.18)$ & $3(8.82)$ \\
Total CRAO & $4(80)$ & $1(20)$ \\
Total patients & $40(90.91)$ & $4(9.09)$ \\
\hline
\end{tabular}

Values are presented as number (\%).

$\mathrm{CRAO}=$ central retinal artery occlusion.

Perfusion recovery was observed in $40(90.91 \%)$ out of the 44 patients. Recovery was observed in $100 \%$ of those in the incomplete stage, $91.18 \%$ in the subtotal stage, and $80 \%$ in the total stage (Table 4).

\section{Safety outcomes}

Following IAT, two patients (4.55\%) developed intracranial hemorrhage, a serious complication. Both were admitted to the neurosurgery clinic of our hospital and underwent the Burr-hole drainage procedure. One of the patients was discharged in a mentally alert state, and the other underwent drainage surgery due to the occurrence of intracranial hemorrhage 8 days after the procedure. However, the guardian of the second patient did not wish for active treatment after the operation and, therefore, the patient died due to a Do-Not-Resuscitate order. Another patient (2.27\%) had a reoccurrence of CRAO in the same eye within 217 days after the procedure, and IAT was administered once more. Initially, this patient was admitted to the hospital with an ART of 36 seconds in the 'VA hand motion' during the first visit, which improved to 12 seconds of ART in the 'VA counting fingers' during the last visit following IAT. The patient complained of decreased VA again after 217 days in the same eye and was diagnosed with a relapse of CRAO after an examination. VA hand motion and ART were measured and found to be $60 \mathrm{sec}$ onds. Therefore, IAT was performed again, where VA counting fingers and ART improved to 16 seconds. Furthermore, dizziness occurred in nine patients (20.45\%), and headache in eight (18.18\%), which included the three patients with dizziness. Two patients $(4.55 \%)$ complained of systemic itching that improved after intramuscular injection of chlorpheniramine maleate $2 \mathrm{~mL}$ (Peniramine;
Table 5. Complication after intra-arterial thrombolysis

\begin{tabular}{lc}
\hline Complication & No. of patients \\
\hline Dizziness & $9(20.45)$ \\
Headache & $8(18.18)$ \\
Intracranial hemorrhage & $2(4.55)$ \\
Itching sensation & $2(4.55)$ \\
Hand tremors & $2(4.55)$ \\
Retinal artery reocclusion & $1(2.27)$ \\
Ocular pain & $1(2.27)$ \\
Tinnitus & $1(2.27)$ \\
Ptosis & $1(2.27)$ \\
\hline
\end{tabular}

Values are presented as number (\%).

Yuhan, Seoul, Korea) and dexamethasone $5 \mathrm{~mL}$ (Dexamethasone; Bukwang, Seoul, Korea). The itch was considered to be a side effect of the contrast. One of these patients $(2.27 \%)$ complained of eye pain, and an ophthalmic examination determined that it was not due to an increase in intraocular pressure. Hand tremors occurred in two patients $(4.55 \%)$ (Table 5), and there were no other ophthalmic complications such as neovascularization or glaucoma.

\section{Discussion}

There has been no treatment established for the management of CRAO, and the use of IAT has been a subject of debate due to the paucity of large-scale clinical trials in this regard. The EAGLE trial had shown similar outcomes for IAT compared to conservative treatments but did not recommend it owing to the high rate of adverse effects [21]. However, the effectiveness of conservative treatment was insufficient. Moreover, IAT was found to be more effective in treating CRAO in the few published case studies and meta-analyses that showed better VA improvements than the conservative treatment $[16,17,24,27,28]$. Therefore, IAT appears to be a good treatment option for CRAO and cannot be overlooked.

CRA is a branch of the ophthalmic artery that originates from the ICA, and occlusion of the CRA or the ophthalmic artery can be considered as an ocular analog of stroke among ICA occlusions [22]. The American Heart Association and the American Stroke Association included retinal vascular infarction as a central nervous systemic infarction 
in 2013 and transient retinal infarction in the category of transient ischemic attack [29]. In addition, Biousse and Trobe [30] suggested that transient monocular visual loss could predict cerebral infarction. Moreover, risk factors in the cardiovascular system, such as hypertension and atherosclerosis, were found in $78 \%$ of the 77 non-arteritic CRAO patients in the EAGLE trial [31]. Moreover, in the current study as well, risk factors in the cardiovascular system were identified in $25(56.82 \%)$ of the 44 participants. In this regard, the examination of similarity between stroke and CRAO indicates that CRAO treatment could also be applied similarly for stroke treatment.

To evaluate the degree of perfusion, ART and AVP can be measured [32], which quantifies the macrocirculation of the retina in both normal and abnormal eyes [33]. ART is the time at which it is first visible in the CRA when fluorescein is injected into the antecubital vein. It reflects the resistance of blood flow in the microcirculation and the pulmonary microcirculation and serves as an indicator of coronary blood flow. AVP is the interval between the first appearance of fluorescein in the retinal artery and its appearance in the corresponding retinal vein. It is an index useful in studying retinal circulation, such as in CRAO, as it helps to estimate the mean blood flow and microcirculation in the retina [34,35]. Pemberton and Britton. [36] reported that although the results of ART are not always consistent, it could provide information that can support or clarify the diagnosis of occlusive cerebral vascular disease. Therefore, since delayed repletion of the retinal artery on FA can be caused by stenosis or occlusion of the ICA and as ART delay itself cannot be considered as a basis for diagnosing CRAO, clinical features such as decreased vision and retinal edema on the fundus should be evaluated in addition to the flow delay when diagnosing CRAO and classifying it into stages [25].

In this study, we divided the patients into three stages based on VA, fundus findings, and FA to determine the efficacy and prognosis of IAT in CRAO. There was a statistically significant difference in baseline BCVA during patient classification ( $p=0.000$ ), prompting us to use BCVA as the criterion for classification of the stages. The initial VA in patients at the subtotal stage was poorer than that in patients at the incomplete stage, while patients at the total stage showed poorer VA than those in the subtotal stage.

Out of a total of 44 patients, IAT was performed in five patients at the incomplete stage (11.36\%), 34 at the subtotal
(77.28\%), and five at the total (11.36\%). Furthermore, VA improved in 31 patients (70.45\%), of whom six (13.64\%) from the subtotal stage showed significant improvement. Anatomical reperfusion was confirmed in 42 patients (95.45\%). Schmidt and Schumacher. [25] also performed IAT by classifying 46 participants into three groups: 10 patients in the incomplete stage (21.74\%), 26 in the subtotal $(56.52 \%)$, and 10 in the total $(21.74 \%)$, similar to the distribution of our study. Furthermore, 28 patients (60.87\%) showed improvement in VA, of whom 11 (23.91\%) showed significant improvement, and most of the VA was maintained for an average of 2.1 years after the procedure. Schmidt and Schumacher. [25] also stated that it is essential to divide CRAO into stages to identify whether IAT is effective in patients classified based on these stages and to ensure that conservative treatment is recommended instead for patients in the incomplete stages. Furthermore, the study reported that IAT must commence within a few hours if the condition were to progress from an incomplete to a subtotal stage. In another study, Ahn et al. [23] performed IAT in 57 patients (13 in the incomplete stage [22.80\%], 28 in subtotal [49.12\%], and 16 in total [28.07\%]) from a total of $101 \mathrm{CRAO}$ patients. Significant VA improvement at the final visit was seen in 21 of the 57 patients (36.84\%), while 45 of 51 patients $(88.24 \%)$ who underwent FA at the last visit showed signs of anatomical reperfusion. Among the patients who showed significant improvement in VA, 11 out of $13(84.62 \%)$ at the incomplete stage showed improvement. Consequently, IAT was considered effective at the incomplete stage, and stage was the only variable that was associated with BCVA after one month. The report also stated that the initial VA and the stages were significantly associated with the final VA.

Thrombolytics are recommended for administration within 4.5 hours of stroke onset [37,38], whereas the appropriate treatment time for CRAO has not been determined yet [4]. The results of experimental studies on rhesus monkeys have been used to estimate human retinal tolerance time where irreversible damage from retinal ischemia was evident 4 hours after occlusion [39,40]. However, since this a result obtained from an animal study, it cannot accurately reflect the situation in humans. Moreover, in a recent meta-analysis, systemic fibrinolysis was reported to have beneficial effects when treatment was provided within 4 to 5 hours of symptom onset [41], and IAT effects were reported to decrease after 6 hours [24]. 
Moreover, a part of the retinal flow remains when CRAO occurs in humans. In the case of an incomplete stage, there is a possibility to observe improvements in the collateral circulation, which results in longer resistance to ischemia. In addition, active treatments such as IAT could help restore the blood supply in the retina, as they have a wider therapeutic window. However, they are less effective in situations such as total occlusion or pronounced hypoperfusion of the choroid [15,22]. Tobalem et al. [42] considered that infarction of the retinal ganglion could occur within 12 to 15 minutes in the complete stage CRAO and reported such reasons as an explanation for the absence of evidence-based treatment for CRAO. Correspondingly, this study also identified the Snellen chart VA of patients in the incomplete stage to be better than $20 / 32$ at the time of visit to the hospital, which did not highlight the effects of IAT. However, patients in this stage showed the best BCVA results, and there were no effects at all in those at the total stage. Another recent meta-analysis also showed significant improvements in VA $(50 \%-76.9 \%)$ in those in the incomplete stage, but this decreased in those in the subtotal stage to $10.6 \%$ to $14.3 \%$, and was $0 \%$ in those in the total stage [24]. If a randomized trial can be conducted to identify the treatment effect of IAT in the future, the average effect could be expected to increase if the total stage group, which shows no treatment effect, is excluded and only those from the incomplete and subtotal stages are included.

In the current study, there was a statistically significant difference in the degree of VA improvement between each of the patient groups that underwent IAT (Table 3) $(p=$ 0.002).

There also was a significant difference in the degree of VA improvement between the incomplete stage and the subtotal stage ( $p=0.005$ ), but no improvements were seen between the incomplete and total stage $(p=0.114)$ as well as between the subtotal and total stage $(p=0.086)$.

This may be due to a good initial VA reading for the incomplete stage, which did not show significant improvement even after treatment. However, in the subtotal stage, the initial VA was poor but had significantly improved due to treatment and, ultimately, showed the highest proportion of VA improvement among the groups. Furthermore, the absence of VA improvement in the total stage, which showed no differences during comparisons, was also considered to have contributed to such results.
Regardless of the CRAO stage, the 29 patients (n1) who visited the hospital with referrals and received treatment at the outpatient clinic, as well as 14 patients who had visited the emergency department for treatment (n2) (total $=43$, one patient was excluded as the they experienced a relapse of CRAO), had significant differences at the time of visit between two groups ( $\mathrm{n} 1=19.810$ hours, $\mathrm{n} 2=4.071$ hours, $p<0.0001$ ).

However, there was no statistically significant difference between the two groups based on the time of the visit and the criteria for improvement of VA outlined in this study ( $p=0.168$ ), and no difference was observed even when the figures were calculated at the absolute value $(p=0.409)$. This indicates that the time of visit as well as the prognosis of VA did not statistically differ, suggesting that IAT may be a method that could improve BCVA and reperfusion in patients with CRAO even if there is a difference in the time of hospital visit.

In this study, we injected the UK into the superselected ophthalmic artery $(n=36)$. However, when there was no possible selection, UK was injected near the entrance of the ophthalmic artery $(n=8)$. There was no significant effect on the prognosis after treatment between these two groups. Selective IAT acts locally on the blocked artery, compared to systemic thrombolysis, and reduces systemic exposure, allowing smaller amounts to be used. However, it may cause intracranial or intraretinal hemorrhage, transient ischemic attack, and other neurological symptoms [5]. Intracranial hemorrhage, in particular, is one of the worse complications, and this occurred in two patients $(4.55 \%)$ in the current study, one of whom died.

The probability of VA recovery following conservative treatment for CRAO is approximately $1 \%$ to $15 \%[9,11,43]$; hence, selective IAT was considered in this study when determining the pattern of VA recovery. The rate of hemorrhagic complications in procedures such as cerebral infarction (IAT on infarct) is approximately $5.5 \%$ to $20 \%$ [44-46], and fibrinolytic therapy can always be accompanied by hemorrhagic complications, among other side effects. However, the treatment's benefits cannot be ignored.

There are a few limitations to our study. First, our retrospective study was performed on all patients except for those who refused to undergo therapy or in those who had received anti-platelet therapy (four patients). Since neither the patients were recruited nor were they examined to write a thesis. Therefore, a control group was not included 
because follow-up would have been not possible or, even if it were possible, could not be conducted consistently. Accordingly, there are also limitations while comparing and predicting the progress, prognosis, and risks associated with IAT treatment. Since the therapeutic window was wide (up to 74.5 hours), the possibility that retinal ischemia had already occurred cannot be dismissed. This is due to the policy of Korea's tertiary hospitals requiring referrals for outpatient treatment but not requiring this when the patient visits through the emergency department. Second, we did not include a limit for BCVA while selecting patient groups. Therefore, patients with good VA were also included, which may have affected the baseline values and the analysis of IAT results. This was done because all the 44 patients, except for one who had a relapse of CRAO, experienced their first visit to the emergency department or underwent treatment at the outpatient department. Moreover, the cilioretinal artery, which is a branch of the posterior ciliary artery, is present in $32 \%$ to $50 \%$ of people $[3,8]$, but not a branch of CRA; hence, it cannot be affected by CRAO as it supplies blood to the papillomacular bundle. Therefore, patients with cilioretinal arteries may have had a better prognosis for VA [5,42]. A total of 11 patients had cilioretinal arteries in this study, of whom five were in the incomplete stage, and six were in the subtotal stage.

Furthermore, when the types were subdivided in a table (Table 1), the CRAO stage appeared to be more prevalent in older patients ( $p=0.069)$, and the average time taken to make the hospital visit appeared to reduce with higher CRAO stages ( $p=0.323)$. Unfortunately, there were no significant differences in these parameters between the three groups. This was a limitation since the number of patients in each group was small, with less than five in each group, particularly in the incomplete and total stages. Hence, data were not normally distributed.

CRAO has a low prevalence (1-1.4 per 100,000 people) and the fact that patients see objects with both eyes might delay symptom recognition. There is also a possibility that hospitalization could be delayed due to the patient's assumption that there will be an improvement after a sudden decrease in VA. Therefore, educating the community regarding these abnormalities as well as stroke symptoms is necessary to instill in people the importance of recognition of the sudden decrease in vision, prompting them to visit an emergency department or a nearby ophthalmology clinic for treatment.
Besides, proper education about the need for regular ophthalmic examination and addressing modifiable risk factors, such as metabolic syndrome, lifestyle, alcohol, and smoking, are necessary for people with at-risk conditions such as hypertension, diabetes, and hyperlipidemia.

In conclusion, CRAO is an ocular emergency that could lead to loss of vision; therefore, other systemic ischemic events must also be considered. In addition to CRA reperfusion, ocular complications and systemic vascular ischemia must also be managed together. In the current study, IAT was effective in improving VA in the subtotal stage of CRAO and was effective for reperfusion in all the stages. Therefore, IAT should be used to treat CRAO with sufficient consideration of the risks and benefits. A better randomized controlled trial will be needed in the future to demonstrate the universally safe and effective use of IAT.

\section{Conflict of Interest}

No potential conflict of interest relevant to this article was reported.

\section{Acknowledgements}

This study was supported by Wonkwang University in 2020.

\section{References}

1. Jusufovic M, Elsais A, Kerty E. Seven points that explain the lack of effect from reperfusion therapy in central retinal artery occlusion. Ophthalmol Retina 2019;3:713-5.

2. Hayreh SS, Zimmerman MB. Central retinal artery occlusion: visual outcome. Am J Ophthalmol 2005;140:376-91.

3. Hakim N, Hakim J. Intra-arterial thrombolysis for central retinal artery occlusion. Clin Ophthalmol 2019;13:2489-509.

4. Chen CS, Lee AW, Campbell B, et al. Efficacy of intravenous tissue-type plasminogen activator in central retinal artery occlusion: report from a randomized, controlled trial. Stroke 2011;42:2229-34.

5. Sharma RA, Dattilo M, Newman NJ, Biousse V. Treatment of nonarteritic acute central retinal artery occlusion. Asia Pac J Ophthalmol (Phila) 2018;7:235-41. 
6. Biousse V, Nahab F, Newman NJ. Management of acute retinal ischemia: follow the guidelines! Ophthalmology 2018;125:1597-607.

7. Gilbert AL, Choi C, Lessell S. Acute management of central retinal artery occlusion. Int Ophthalmol Clin 2015;55:157-66.

8. Varma DD, Cugati S, Lee AW, Chen CS. A review of central retinal artery occlusion: clinical presentation and management. Eye (Lond) 2013;27:688-97.

9. Rumelt S, Dorenboim Y, Rehany U. Aggressive systematic treatment for central retinal artery occlusion. Am J Ophthalmol 1999;128:733-8.

10. Fraser SG, Adams W. Interventions for acute non-arteritic central retinal artery occlusion. Cochrane Database Syst Rev 2009;2009:CD001989.

11. Atebara NH, Brown GC, Cater J. Efficacy of anterior chamber paracentesis and Carbogen in treating acute nonarteritic central retinal artery occlusion. Ophthalmology 1995;102:2029-34.

12. Agarwal N, Gala NB, Baumrind B, et al. Endovascular management of central retinal arterial occlusion. Vasc Endovascular Surg 2016;50:579-81.

13. Dumitrascu OM, Shen JF, Kurli M, et al. Is intravenous thrombolysis safe and effective in central retinal artery occlusion? A critically appraised topic. Neurologist 2017;22: $153-6$.

14. Preterre C, Godeneche G, Vandamme X, et al. Management of acute central retinal artery occlusion: intravenous thrombolysis is feasible and safe. Int J Stroke 2017;12:7203.

15. Schmidt DP, Schulte-Monting J, Schumacher M. Prognosis of central retinal artery occlusion: local intraarterial fibrinolysis versus conservative treatment. AJNR Am J Neuroradiol 2002;23:1301-7.

16. Arnold M, Koerner U, Remonda L, et al. Comparison of intra-arterial thrombolysis with conventional treatment in patients with acute central retinal artery occlusion. $J \mathrm{Neu}$ rol Neurosurg Psychiatry 2005;76:196-9.

17. Richard G, Lerche RC, Knospe V, Zeumer H. Treatment of retinal arterial occlusion with local fibrinolysis using recombinant tissue plasminogen activator. Ophthalmology 1999;106:768-73.

18. Pettersen JA, Hill MD, Demchuk AM, et al. Intra-arterial thrombolysis for retinal artery occlusion: the Calgary experience. Can J Neurol Sci 2005;32:507-11.

19. Weber J, Remonda L, Mattle HP, et al. Selective intra-arte- rial fibrinolysis of acute central retinal artery occlusion. Stroke 1998;29:2076-9.

20. Feltgen N, Neubauer A, Jurklies B, et al. Multicenter study of the European Assessment Group for Lysis in the Eye (EAGLE) for the treatment of central retinal artery occlusion: design issues and implications. EAGLE Study report no. 1: EAGLE Study report no. 1. Graefes Arch Clin Exp Ophthalmol 2006;244:950-6.

21. Schumacher M, Schmidt D, Jurklies B, et al. Central retinal artery occlusion: local intra-arterial fibrinolysis versus conservative treatment, a multicenter randomized trial. Ophthalmology 2010;117:1367-75.el.

22. Biousse V, Calvetti O, Bruce BB, Newman NJ. Thrombolysis for central retinal artery occlusion. $J$ Neuroophthalmol 2007;27:215-30.

23. Ahn SJ, Kim JM, Hong JH, et al. Efficacy and safety of intra-arterial thrombolysis in central retinal artery occlusion. Invest Ophthalmol Vis Sci 2013;54:7746-55.

24. Page PS, Khattar NK, White AC, et al. Intra-arterial thrombolysis for acute central retinal artery occlusion: a systematic review and meta-analysis. Front Neurol 2018;9:76.

25. Schmidt D, Schumacher M. Stage-dependent efficacy of intra-arterial fibrinolysis in central retinal artery occlusion (CRAO). Neuroophthalmology 1998;20:125-41.

26. Lange C, Feltgen N, Junker B, et al. Resolving the clinical acuity categories "hand motion" and "counting fingers" using the Freiburg Visual Acuity Test (FrACT). Graefes Arch Clin Exp Ophthalmol 2009;247:137-42.

27. Noble J, Weizblit N, Baerlocher MO, Eng KT. Intra-arterial thrombolysis for central retinal artery occlusion: a systematic review. Br J Ophthalmol 2008;92:588-93.

28. Beatty S, Au Eong KG. Local intra-arterial fibrinolysis for acute occlusion of the central retinal artery: a meta-analysis of the published data. Br J Ophthalmol 2000;84:914-6.

29. Sacco RL, Kasner SE, Broderick JP, et al. An updated definition of stroke for the 21st century: a statement for healthcare professionals from the American Heart Association/ American Stroke Association. Stroke 2013;44:2064-89.

30. Biousse V, Trobe JD. Transient monocular visual loss. Am J Ophthalmol 2005;140:717-21.

31. Callizo J, Feltgen N, Pantenburg S, et al. Cardiovascular risk factors in central retinal artery occlusion: results of a prospective and standardized medical examination. Ophthalmology 2015;122:1881-8.

32. Kim HM, Park YJ, Park KH, Woo SJ. Visual field defects 
and changes in central retinal artery occlusion. PLoS One 2019;14:e0209118.

33. Cekic O, Keserci B, Ohji M, et al. Macular translocation surgery and retinal circulation times. Retina 2004;24:51-6.

34. Kang G, Lee J. Retinal circulation times in branch retinal vein occlusion. Korean J Ophthalmol 1995;9:107-10.

35. Sun G, Liu X, Gao L, et al. Automatic measurement of global retinal circulation in fluorescein angiography. $J$ Biomed Opt 2018;23:1-8.

36. Pemberton JW, Britton WA. The arm-retina circulation time. Arch Ophthalmol 1964;71:364-70.

37. Demaerschalk BM, Kleindorfer DO, Adeoye OM, et al. Scientific rationale for the inclusion and exclusion criteria for intravenous alteplase in acute ischemic stroke: a statement for healthcare professionals from the American Heart Association/American Stroke Association. Stroke 2016;47: 581-641

38. Powers WJ, Rabinstein AA, Ackerson T, et al. Guidelines for the early management of patients with acute ischemic stroke: 2019 update to the 2018 guidelines for the early management of acute ischemic stroke: a guideline for healthcare professionals from the American Heart Association/American Stroke Association. Stroke 2019;50:e344418.
39. Hayreh SS, Zimmerman MB, Kimura A, Sanon A. Central retinal artery occlusion. Retinal survival time. Exp Eye Res 2004;78:723-36.

40. Hayreh SS. Acute retinal arterial occlusive disorders. Prog Retin Eye Res 2011;30:359-94.

41. Schrag M, Youn T, Schindler J, et al. Intravenous fibrinolytic therapy in central retinal artery occlusion: a patient-level meta-analysis. JAMA Neurol 2015;72:1148-54.

42. Tobalem S, Schutz JS, Chronopoulos A. Central retinal artery occlusion: rethinking retinal survival time. $B M C O p h-$ thalmol 2018;18:101.

43. Schmidt D, Schumacher M, Wakhloo AK. Microcatheter urokinase infusion in central retinal artery occlusion. Am J Ophthalmol 1992;113:429-34.

44. Furlan A, Higashida R, Wechsler L, et al. Intra-arterial prourokinase for acute ischemic stroke. The PROACT II study: a randomized controlled trial. Prolyse in Acute Cerebral Thromboembolism. JAMA 1999;282:2003-11.

45. Kase CS, Furlan AJ, Wechsler LR, et al. Cerebral hemorrhage after intra-arterial thrombolysis for ischemic stroke: the PROACT II trial. Neurology 2001;57:1603-10.

46. Chalela JA, Katzan I, Liebeskind DS, et al. Safety of intra-arterial thrombolysis in the postoperative period. Stroke 2001;32:1365-9. 